\title{
Diskursive Problemermittlung statt linearer Wissensvermittlung - am Beispiel des Sahelnomadismus
}

\section{Problembeschreibung}

* Bergrutschkatastrophe 1987 im Veltlintal: Der Schweizer Sekretär der Stiftung für Alpine Forschung, Fritz Hans Schwarzenbach, sieht seine jahrelangen Warnungen vor einer Übernutzung der Alpen, vor allem durch den Bergtourismus, bestätigt. Der Schweizer Geograph Thomas Mosimann macht hingegen darauf aufmerksam, daß die stärkste Bergrutschgefahr in touristisch noch unerschlossenen Tälern bestehe. (FRANKFURTER RUNDSCHAU v. 10.8.1987).

* Hochwasserkatastrophe 1988 im Bereich der Flußsysteme der Bundesrepublik Deutschland: Der Wissenschaftliche Beirat der Bundesregierung führt dieses Ereignis auf einmalig heftige Niederschläge im Oberlaufbereich der großen Flüsse zurück. Dem widerspricht der Geobotaniker Emil Dister durch Verweis auf die in immer kürzeren Abständen und unabhängig von der jeweiligen Niederschlagshöhe sich wiederholenden Überschwemmungen (DER SPIEGEL Nr. 14/1988, S. 106 f.).

* «Industriestandort Bundesrepublik in Gefahr?»so lautet eine in den Medien wiederholt aufgeworfene Frage, die auch in Schulbüchern abgehandelt wird: Mit Ja wird sie seitens der Arbeitgeberverbände beantwortet - denn die Arbeit sei zu teuer; verneint wird sie hingegen von den Gewerkschaften - allerdings müßten Binnenkaufkraft und Investitionen gesteigert werden.

* Thema Entwicklungsprobleme: Als Haupterklärungsmoment bei der Suche nach Ursache und Lösung der Überlebensprobleme in Entwicklungsländern wird immer wieder auf die Bevölkerungsexplosion hingewiesen. Als Problemursache wird sie von den meisten Geographen, als Problemfolge von der Mehrzahl der Sozialwissenschaftler gewertet.

* Problem Unterentwicklung: In der Alltagswahrnehmung scheinen sich Armut und Verelendung in der Dritten Welt hinreichend durch den «Teufelskreis der Armut» zu erklären. Kritische Wissenschaftler sehen in diesem jedoch lediglich ein Symptom. Bei der Ursachensuche gehen sie von strukturell gewordenen Behinderungen aus, die historisch und gesellschaftswissenschaftlich zu ermitteln seien.

Diese - mühelos erweiterbaren - Szenarien divergierender Probleminterpretationen deuten das un- geheure didaktische Potential an, das den meisten erdkundlichen Themen zu eigen ist. Ist das Ziel des Erdkundeunterrichtes nicht «eine Propädeutik dessen, was an der Universität unter Geographie verstanden wird», sondern geht es vielmehr darum, «Schüler instand(zu)setzen, ihre jetzigen und künftigen „räumlichen Lebenssituationen” intensiver wahrzunehmen und nach Vorzügen und Defiziten, Erhaltungs- und Veränderungswürdigkeit kritisch zu bedenken" (HARD 1982, S. 284, Hervorhebung WSW), dann wäre zweifelsohne eine der jeweiligen Komplexität und Widersprüchlichkeit angemessene diskursive Themenbehandlung angebracht.

Betrachtet man Schulbücher der neueren Generation danach, wie sie mit Inhalten verfahren, fehlt nicht nur jegliche Auseinandersetzung zwischen unterschiedlichen Deutungsmustern, sie sind durchwegs Aneignungsprinzipien verpflichtet, die einer diskursiven Lernweise hinderlich sind:

1. Inhalte und Aufgabenstellungen sind auf passives, nachvollziehendes Lernen, auf die Rezeption von Vorgedachtem festgelegt. Infolge des jedem Thema zugebilligten äußerst knappen Raumes sind die Schulbuchinformationen extrem ausgedünnt und komprimiert. Für «Überflüssiges», für - neben Wichtigem -auch weniger Bedeutsames ist kein Platz, noch weniger aber für Abweichendes, Widersprüchliches. Solcherart «geglättete» Informationen strotzen - wie könnte es anders sein - von impliziten Wertungen und Bewertungen, allein: Diese werden dem Schüler nicht kenntlich gemacht. Bezeichnenderweise bremst der Zwang zur Informationsbeschränkung den wissenschaftsgeschichtlich verständlichen Hang zur Vollständigkeit keineswegs. Der Kieler Erziehungswissenschaftler Jochen Grell hat - explizit unter Bezugnahme auf Erdkundebücher - festgestellt: "Sie erwecken den Eindruck, als stellten sie alles dar, was über das betreffende Sachgebiet bekannt ist . . . Ich fürchte, daß so etwas stumpf macht. Zwar ist in den Büchern bei-

W. Schmidt-Wulffen, Prof., Dr., Universität Hannover, FB Ew I, Geographie und ihre Didaktik, Bischofshofer Damm 76, D-3000 Hannover 1 
nahe jedes denkbare Thema erwähnt, aber dafür beinahe keines richtig und gründlich und seiner Bedeutung entsprechend. Die Bücher sind so sachlich, $\mathrm{da} ß$ es große oder kleine Themen nicht mehr gibt. Alles wird gleich wichtig oder unwichtig. Das ist Ausgewogenheit» (GRELL 1984, S. 49).

2. Inhalte werden nicht nur ausgedünnt, sondern zwecks Komplexitätsreduktion auch qualitativ, d. h. in ihrem Gehalt gemindert. Die eigentlich erst durch ihre gesellschaftliche Brisanz als Lehrbeispiele didaktisch fruchtbaren Themen werden in ein inkonsistentes didaktisches Strukturierungsschema gepreßt und gehen dadurch ihres gesellschaftlichen Erkenntnisgehaltes verlustig, etwa wenn gesellschaftliche Realität «sozialgeographisch», «allgemeingeographisch» oder «kulturökologisch» interpretiert wird (zu den Schwächen des sozialgeographischen Konzeptes der Münchener Schule vgl. WERLEN 1987, S. 231-252, denen der Allgemeinen Geographie nach A. Schultze vgl. SCHMIDT-WULFFEN 1982, S. 15-20, denen des kulturökologischen Mensch-Natur-Paradigmas DAUM/SCHMIDT-WULFFEN 1980, S. 63-83).

3. Die didaktische Dürftigkeit spiegelt die geringe Neigung vieler Fachdidaktiker, sich ernsthaft auf sozialwissenschaftliche Fragestellungen und Theorien einzulassen (ein aus dem Werdegang als Lehrer mit Beifächern wie Sport, Englisch oder Mathematik durchaus erklärliches Verhalten). Die Münchener Politologin E. MICHEHELLES hat kürzlich auf die Konsequenzen sozialwissenschaftlicher Abstinenz in Blick auf die Afrikakapitel bundesdeutscher Erdkundebücher hingewiesen: «Während in den Sozialwissenschaften davon ausgegangen werden kann, daß zur Erklärung gewisser Erscheinungen zum Teil widersprüchliche Interpretationen zur Verfügung stehen, sind in den Erdkundeschulbüchern alternative Deutungsmuster nicht enthalten» (1985, S. 9).

4. Problemverluste erklären sich nicht allein aus rezipierenden Lernweisen, Komplexitätsreduktion und sozialwissenschaftlichen Unterlassungen, sondern stehen auch in Zusammenhang mit dem über Lehrpläne bürokratisch verordneten Zwang, Lernen auf abfragbare (Fach)Begriffe - als dessen wesentlichstes Ergebnis - auszurichten. Solche Begriffe beanspruchen damit den Rang einer Tatsache. Der Wissenschaftstheoretiker Ludwig Fleck erläutert den Zusammenhang zwischen Begriffslernen und damit einhergehender Bildung von Werturteilen sehr treffend: «Begriffe müssen den Schülern so als etwas Feststehendes, Bleibendes, vom subjektiven Meinen des Forschers Unabhängiges erscheinen. Damit findet eine naive Wertung statt, die sich durch die Verflachung ihrer Ergebnisse rächt» (FLECK 1935/1980, S. 1). Und der Hamburger Erziehungswissenschaftler Wolfgang Schulz bestätigt dieses Urteil: «Ich halte es für einen schrecklichen Irr- tum zu glauben, daß man auch in einer sich wandelnden Welt den Nachwachsenden die Werte, Handlungsmuster und Informationssysteme zunächst einmal als ehern und unbezweifelbar hinstellen müsse, weil sie sie sonst nicht ernsthaft aufnähmen... Die Schule wird unglaubwürdig (gegenüber außerschulischen Informationsträgern), wenn sie die Wahrheit zurückhält . . . Deshalb wird es, so hoffe ich, bald zum Kanon aller Lehrpläne und Schulbücher gehören, diese Pluralität, diese Widersprüchlichkeit der gesellschaftlichen Realität mit abzubilden» (schULZ 1984, S. 44).

Ehe eine Gegenposition umrissen wird, soll am Thema «Nomadismus im Sahel» die typische Inhaltsdarstellung in Schulbüchern umrissen und mit den Ergebnissen sozialwissenschaftlicher Forschung konfrontiert werden. Aus dieser - notgedrungen kursorischen - Darstellung lassen sich Rückschlüsse auf die Berechtigung der hier vorgenommenen Kritik ziehen, aber auch Schlußfolgerungen für eine alternative Vermittlungsweise.

\section{Der Sahelnomadismus in Forschung und Schulbuch}

Die Analyse der sich verschlechternden Lebenssituation der Sahelnomaden, wie sie in der Geographie vor allem von $\mathrm{H}$. Mensching und F. Ibrahim vorgelegt wurde (vgl. z. B. MENSCHING 1986), sieht in den Naturbedingungen («Variabilität der Niederschläge») eine relative Konstante («Dürren hat es schon immer gegeben»). Die sich in den letzten Jahrzehnten dramatisch verschlechternde Lebenssituation der Nomaden ist somit nicht auf eine Klimaanomalie oder einen möglicherweise zwar vorhandenen, aber nur langfristig wirksam werdenden Klimawandel zurückzuführen, sondern auf das Verhalten der Menschen. Die verringerten Lebenschancen der Nomaden werden vor allem an den landschaftlichen Zerstörungen («Desertifikation») ablesbar, die die Existenzgrundlage der Viehhalter zunehmend einschränken. Da dem Klima nur eine auslösende Funktion zufällt, ist das Verhalten der Menschen bereits durch diesen Umstand eindeutig als ein Fehlverhalten ausgewiesen:

- Es werden zu große Herden gehalten (aus Prestigegründen)

- Bäume werden bedenkenlos abgeholzt (zur Futter- und Brennholzgewinnung)

- Die Bevölkerung ist sprunghaft angewachsen (infolge medizinischer Maßnahmen)

- Die Nomaden sind in Ungunstgebiete abgedrängt worden (durch nach Norden vorrückenden Ackerbau).

Das Haupterkenntnisinteresse richtet sich auf ökologische Wirkungsmechanismen; Maßstab der Beur- 
teilung des «Fehlverhaltens» ist das ökologische Gleichgewicht - nicht aber eine soziale Kategorie, etwa die auf Überleben gerichteten Handlungen der Nomaden. Da die Natur nur über den Menschen «repariert» werden kann, fällt ihm bei allen Lösungskonzepten eine Schlüsselrolle zu. Da aber für dessen Handlungsmotive, so sie über vordergründige Erklärungen hinausreichen (wie Prestigestreben, traditionelles Verhalten), kein Erklärungsbedarf gesehen wird, da nicht gefragt wird, warum die Nomaden sich so verhalten, wie sie sich verhalten, werden Lösungsvorschläge gemacht, bei denen die Betroffenen keine aktive Rolle spielen. Sie können überdies nur längerfristig realisiert und - sofern überhaupt - auch nur längerfristig wirksam werden. Solche Maßnahmen reichen vom Aufbau von Frühwarnstationen, einer Verbesserung von Infrastruktur und Viehvermarktungssystemen über die Einrichtung einer kontrollierten Weidewirtschaft bis hin zu Familienplanung und zur Rückverlagerung des Ackerbaus nach Süden. Solche Konzepte mit passiver Rollenzuweisung für die Nomaden scheinen die Entbehrlichkeit ethnosoziologischen Handlungswissens zu bestätigen. Durch seine Geschlossenheit scheint dieser Erklärungs- und Lösungsansatz in sich plausibel.

Im Bereich der Sozialwissenschaften haben sich vor allem Entwicklungs- und Ethnosoziologen mit dem Sahelnomadismus auseinandergesetzt. Infolge einer - historische Prozesse einbeziehenden - Verhaltensanalyse, die auf der Mikro-Ebene ansetzt und die Verflechtungen mit der Außenwelt rekonstruiert, sind sie zu tiefergreifenden Verhaltenserklärungen als die Geographen gekommen. Im Licht dieser Ergebnisse muß das Verhalten der Nomaden einer Neubewertung unterzogen werden .

Die Soziologen setzen nicht an den landschaftlichen Zerstörungen an, sondern an verlorengegangenen Selbststeuerungsfähigkeiten. Ihr Erkenntnisinteresse richtet sich nicht auf das Funktionieren physischer Umweltmechanismen, sondern auf das sozialer Regelungsmechanismen. Diese dienen dem Ziel der Existenzsicherung («Reproduktion»), denn angesichts des Klimarisikos ist die Existenz besonders in Trocken- und Dürrezeiten gefährdet. Dann sind Biomassen- und Milchproduktion gering, dann müssen zirka $50 \%$ des jährlichen Nahrungsbedarfs von außen gedeckt werden. Dieses Ziel wurde früher über ein Bündel integrierter materieller und sozialer Verhaltensweisen zu erreichen versucht: Durch räumliche Mobilität, eine vielfältige Herdenzusammensetzung (zur Risikominderung und optimalen Ressourcennutzung), durch Sklavenhaltung (zu Ernteabgaben verpflichteter Oasenbauern), durch Transport- und Handelsaufgaben (Karawanenhandel), durch soziale Sicherungen (z.B. Heiratsverbindungen, Viehtausch und -leihe: Die Fähigkeit hierfür wurde mit Prestige belohnt, nicht das Halten einer großen Herde an sich). Im Zuge der in der Kolonialzeit eingeleiteten, danach aber beschleunigt vorangetriebenen Modernisierung sind den Nomaden alle Existenzstützen weggebrochen - Mobilität, Sklavenhaltung, Karawanenhandel - oder diese sind funktionslos geworden, weil sie der uneingeschränkten Mobilität bedürfen: Viehleihe oder Heiratsverbindungen über große Entfernungen. Geblieben ist den Nomaden nur ein «Standbein»: Die Viehhaltung, die für sich nicht einmal reproduktionssichernd war. Daher mußten die Viehbestände vergrößert werden. Neue Belastungen kamen hinzu: Der Steuerzahlung verpflichtet, wurden die Nomaden der Geldwirtschaft unterworfen. Frühere Tauschwerte zwischen Getreide und Fleisch galten nicht länger. In Dürrezeiten stieg der Getreidepreis enorm, während das Vieh von eklatantem Preisverfall betroffen war (in Mali stieg 1984 der 100-kg-Sack Hirse von 60 auf 180 DM, während der Wert eines Rindes von 180 auf 8 DM fiel). Die Grenzziehung verbaute den Nomaden die Möglichkeit, ihr Vieh zu den Märkten zu treiben, die die besten Preise erbrachten. Bei Landreformen wurden z. B. frühere Sklaven mit Bewässerungsland bedacht, während die ethnisch und kulturell als Fremdkörper betrachteten Nomaden von den Staatsführungen übergangen wurden. Während ihnen somit die traditionellen Ressourcen entglitten, blieb ihnen der Zugang zu neuen Lebensmöglichkeiten versperrt. Die weitere Herdenaufstockung, verbunden mit der Überausbeutung der Natur, war die unvermeidbare Konsequenz.

Denkt man nun an Problemlösungen, wird deutlich, daß die von Geographen favorisierten Maßnahmen kaum greifen dürften, da sie die unmittelbaren Existenzprobleme nicht im Visier haben. Unter dem Primat kurzfristiger Überlebenssicherung stehende Menschen können sich den Luxus der ihnen zugemuteten Ressourcenschonung nicht erlauben, deren Früchte erst langfristig wirksam werden können. Hilfe kann kaum erfolgversprechend sein, solange sie nur unserer Rationalität folgt, die subjektiven Handlungsmöglichkeiten und -zwänge aber unberücksichtigt bleiben und Lösungsvorschläge nicht von den Betroffenen auf soziale Erreichbarkeit und die Möglichkeit geprüft werden können, diese selbst zu steuern.

Ein Blick in ein beliebiges Schulbuch genügt, um festzustellen, daß das geographische Deutungsmuster einschränkungslos triumphiert: Da «vergröBern die Hirtenstämme ständig ihre Herden, vor allem wegen des damit verbundenen Ansehens», da «verführten die Jahre mit reicherem Niederschlag die Nomaden zu leichtfertigem Handeln»... . Der Tenor ist eindeutig: Die Nomaden sind nicht die Opfer - das ist die Natur -, sie sind die Täter und Verantwortlichen. Denn sie handeln «traditionell», «leichtfertig», «aus Unwissen» oder «in falschem Glauben». Solcherart Bewertungen sollten nicht vorschnell als rassistisch eingestuft werden; sie spie- 
geln lediglich (?) einen patriarchalisch-eurozentrischen Standpunkt: Sie vermitteln die Gewißheit, im Besitz des einzig gültigen Wertmaßstabes zu sein.

\section{Konsequenzen: Diskursives Lernen}

Vergleicht man den Desertifikationsansatz - das konkurrenzlos herrschende geowissenschaftliche Eigengewächs - mit der sozialwissenschaftlichen Interpretation, dürfte letzterer die größere Plausibilität und eine Überlegenheit an «Tiefgang» und «Reichweite» kaum abzusprechen sein. Dennoch wäre nicht viel gewonnen, würde man eine Deutung durch die andere ersetzen. Wie Geographen und Fachdidaktiker, so sind auch Schüler daran gewöhnt, im Rahmen des Mensch-Natur-Paradigmas zu denken und die gesellschaftliche Vermittlung zwischen Mensch und Natur auszublenden. Gleiches gilt für das Urteil aufgrund eigener Wertprämissen.

Daher bedarf es aller Bemühungen,

* das «traditionelle», d. h. das eindimensionale Mensch-Natur-Denken - weil es in unserer Gesellschaft überbesetzt ist - abzubauen,

* das unreflektierte Denken «von uns aus» in seiner Fragwürdigkeit offenbar werden zu lassen,

* die Grundsätzlichkeit gesellschaftlicher Vermittlung zwischen Mensch und Natur herauszuarbeiten (nicht zuletzt, um für differenziertere Erklärungsmuster auch bei anderen Umweltthemen zu sensibilisieren),

* Schülern die tatsächlichen Abläufe aus den Sinnund Bedeutungszusammenhängen der Betroffenen zu erschließen, um ihnen zu ermöglichen, unter Einschluß der jeweiligen Handlungsspielräume und -zwänge «von den anderen her» zu denken.

Aus diesen Gründen sollte es unstrittig sein, Schülern die Möglichkeit zu verschaffen, sich an konkurrierenden Erklärungsansätzen «abzuarbeiten». Hierfür spricht aber noch eine weitere Überlegung: Es geht nicht um die Ausschaltung eines der beiden «Modelle», sondern um die «dialektische Vermittlung» von Widersprüchlichem, «zu zeigen, daß beide Behauptungen in einer gewissen Weise berechtigt sind, das heißt, ein ,,Moment von Wahrheit" enthalten... Die nähere Analyse widersprüchlicher Aussagen führt zur differenzierteren Beurteilung beider Behauptungen, das heißt zur Präzisierung beider Aussagen und damit zur Beseitigung des Widerspruchs» (HELlBerger 1984, S. 122 f. vgl. auch das folgende Schema).

Diskursives Lernen - das meint: Von einer Vorstellung zur anderen voranzuschreiten, durch Erörtern und Argumentieren. Dabei sollte von den geläufigen Auffassungen ausgegangen werden. Deren Begrenztheit gilt es zu verdeutlichen, deren Vorläufig-

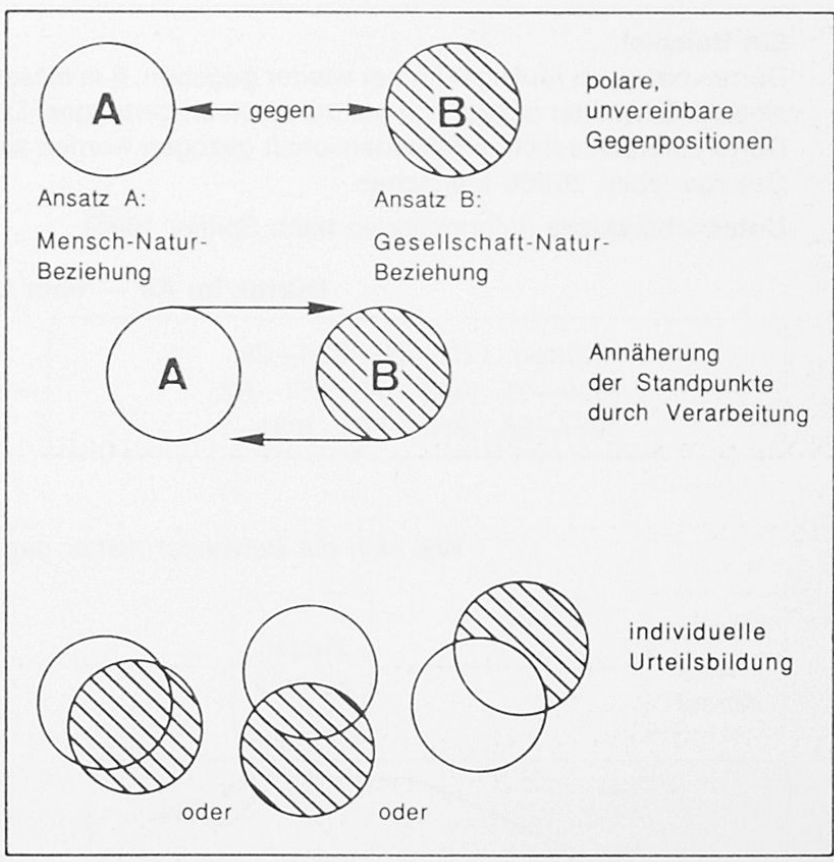

Abb. 1: Organisation des diskursiven Lernprozesses

Lernen als «dialektische Vermittlung» (aus SCHMIDT-WULFFEN 1986)

keit und Unvollkommenheit bewußtzumachen. Neue Informationen, ein neuer Zugang stellt das bisher Angenommene in Frage, treibt den Lernprozeß voran. Dabei sind folgende Regeln zu beachten: Die Materialien, Faktoren, Informationen usw. sind so aufzubereiten,

- daß ein kritischer Umgang des Schülers mit alternativen bzw. kontroversen Denk- und Argumentationsmustern erfolgt und eine Verortung der Positionen möglich wird;

- daß eine Auseinandersetzung des Schülers mit den Argumenten stattfinden kann, die für die öffentliche und für die wissenschaftliche Diskussion bedeutsam sind, auch wenn sie ihm zunächst fremd, ja unverständlich anmuten mögen, um sie dann an den von ihm übernommenen oder verinnerlichten Argumenten zu messen;

- daß die Auseinandersetzung ohne Überforderung erfolgt, d. h. daß sie bei den sozialen Eigenerfahrungen ansetzt und diese ernst nimmt («Schüler dort abholen, wo sie stehen»);

- daß das Fairneßgebot gegenüber Schülern beachtet wird, indem Lehrer ausgewogen - dabei aber dennoch standpunktbezogen - informieren;

- daß unter Einbezug der vorhandenen Informationen ein zwar begründetes, aber doch individuelles Urteil gestattet wird, ohne auf oft fragwürdigen, weil verkürzenden Lernzielen zu beharren;

- daß eine relative Ergebnisoffenheit gewährleistet wird: In der Auseinandersetzung zwischen tiefverwurzeltem und neuem Denken müssen nicht alle Schüler zum gleichen Erkenntnisziel gelangen. 


\section{Ein Beispiel...}

Dürren hat es im Air/Niger immer wieder gegeben, 8 in diesem Jahrhundert. Aber im Unterschied zu anderen Sahelregionen sind die Bewohner erstaunlich gut mit innen umgegangen. Die Touareg des Air verfügen über vier ,,Standbeine“, die bei jeder Dürre unterschiedlich in Mitleidenschaft gezogen worden waren. Nie waren alle „Standbeine“" zugleich betroffen!... Im AirGebirge leben 26000 Menschen

Unterrichtsskizze (Informationen nach Spittler 1985):

\section{Dürren im Air - aber keine Hungerkatastrophen!}

\begin{tabular}{|c|c|}
\hline Dürren: $1911-14,1926-28$, \\
$1930-31,1940-44,1951-52$, \\
$1957-58,1969-1974,1981-?$
\end{tabular}$\quad \longrightarrow \quad \begin{gathered}\text { Natürliche Bedingungen } \\
\text { im Vergleich zu einer } \\
\text { anderen Sahelregion ermitteln... }\end{gathered}$

Wie sich die Bewohner bisher gegenüber der Dürre behaupten konnten:

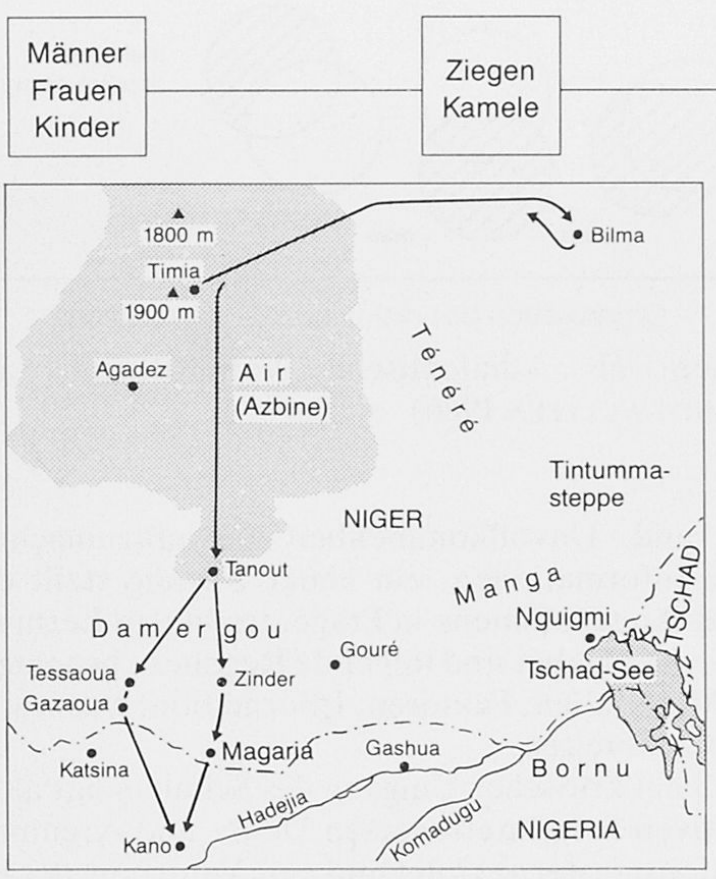

\begin{tabular}{|c|c|}
\hline Gartenwirtschaft: & $\begin{array}{l}\text { Bewässerung } \\
\text { Grundwasserbrunnen } \\
\text { (Gemüse) }\end{array}$ \\
\hline Weidewirtschaft: & $\begin{array}{l}\text { Ziegenhaltung } \\
\text { in den Airtälern (Milch, Käse) }\end{array}$ \\
\hline Kamelhaltung: & $\begin{array}{l}\text { Juli-September } \\
\text { Regenzeit im Air, } \\
\text { Dezember-Februar } \\
\text { Nordnigeria } \\
\text { April-Juni mit Futtervorräten } \\
\text { nach Bilma u. zurück }\end{array}$ \\
\hline Karawanenhandel: & $\begin{array}{l}\text { Hirse nach Bilma, } \\
\text { Salz u. Datteln aus Bilma, } \\
\text { Hirse aus Kano/Nigeria } \\
\text { für die Familie und den Salz- } \\
\text { handel }\end{array}$ \\
\hline
\end{tabular}

Abb. Die Karawanenhandelszüge der Kel Ewery Tuareg aus dem Air (Quelle: Spittler in Journal für Geschichte)

1. Aufgabe: Setzt die vielen kleinen ,,Informationshappen“ zu einem Ganzen zusammen. Versucht damit zu klären, wie die Airbewohner ihr Leben fristen und ihr Überleben bei Dürren sichern konnten.

Zusatzinformationen: In Nigeria gibt es kein Salz, dies ist aber zum Leben wichtig, daher wertvoll. In Bilma gibt es kein Getreide, es ist daher dort sehr teuer. In Nordnigeria ist im Dezember Erntezeit. Dann ist Hirse am billigsten. Während der Trockenzeit, also im Mai/Juni, ist Hirse knapp und teuer, besonders in Bilma.

2. Aufgabe: Klärt, Unter welchen Bedingungen sich Hunger entwickeln wird!

Informationen: 1984 waren 90 \% der Kamele zu schwach für den Karawanenhandel. Um das Verhungern abzuwenden, sollte über amerikanische Entwicklungshilfe der klima- und nahrungsunabhängige LKW-Transport eingeführt werden. Über die deutsche Entwicklungshilfe wurde hingegen lediglich einmalig Salz aus Bilma mit dann wieder abgezogenen LKWs zu den Siedlungen der Touareg geschafft. Dorthin hatten die LKWs auf dem Hinweg Kamelfutter mitgenommen. 
2. Aufgabe: Was könntet Ihr als sinnvolle Lösung vorschlagen und begründen?

Zusatzinformationen: Die Entwicklungshilfe stellt 500'000.- zur Verfügung.Mercedes erklärt sich bereit, Niger zur Bekämpfung des Hungers 10 LKW zu schenken!

Ergebnisse (aus Unterricht mit Schülern und Lehrern in der Lehrerfortbildung):

1. Leistungsfähigere LKW einsetzen, aber für Service, Ersatzteile und Sprit sorgen.

2. LKW verkaufen, den Erlös für Ankauf von Nahrungsmitteln für die Touareg einsetzen.

3. LKW verkaufen, den Erlös für Kamelfutter und gesunde Jungtiere einsetzen.

4. LKW verkaufen, mit dem Erlös die Umsiedlung in den feuchteren Süden bezahlen.

5. Bewässerungssystem instandsetzen, LKW für einmaligen Einsatz benutzen: Getreide und Kamelfutter ins Air transportieren, mit einem Teil des Getreides nach Bilma weiterfahren, von dort Salz ins Air mitnehmen, anschliessend nehmen die Kamele den Transport wieder auf.

Nur die 5. Lösung ermöglicht den Touaregfamilien die Aufrechterhaltung ihrer Existenz. Dieser Vorschlag entspricht der tatsächlich durchgeführten Lösung.

Abb. 2b: Vorschlag einer diskursiven Aufgabenlösung am Beispiel der 2. Aufgabe aus Abb. 2a.

Abb. 3: weiteres Beispiel einer diskursiven Unterrichtsgestaltung

\section{Tiere der Sahelzone}

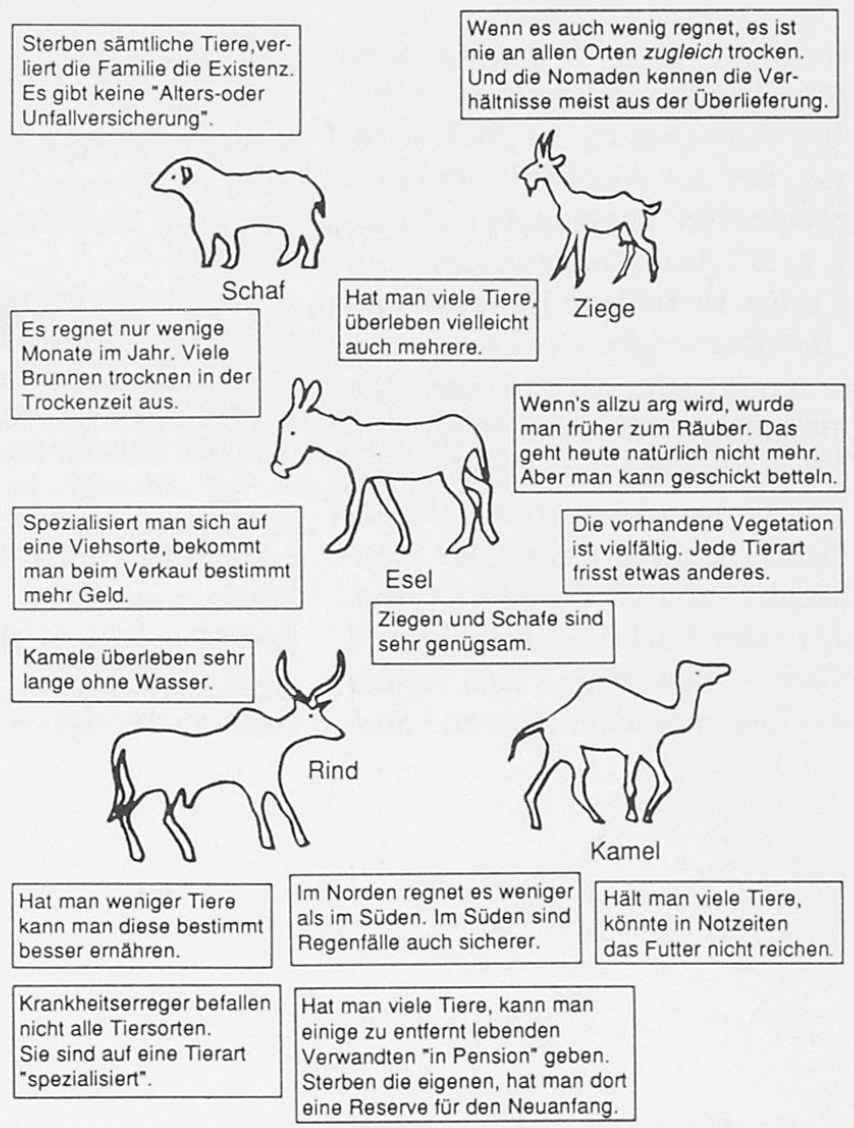

Wenn man Familienmitglieder hat, die sich in der Natur auskennen, kann man wilde

Früchte, Wurzeln usw. sammeln als Notreserve.
Im Süden wohnen Bauern. Die fürchten, dass ihre Felder von den Tierherden zertrampelt werden. 


\section{Diskursives Lernen in der Alltagspraxis}

Das Konzept diskursiven Lernens kann aus Platzgründen hier weder vollständig begründet noch samt den damit verbundenen methodischen Vorgehensweisen ausgebreitet werden (vgl. dazu SCHMIDT-WULFFEN 1985, S. 163-181 und 1986). Daher muß es gegen einige mögliche Mißverständnisse geschützt werden:

- Selbstverständlich ist nicht jeder Unterricht diskursiv zu gestalten; es gibt durchaus auch eine Menge unstrittiger Wissensbestände.

- Diskursives Lernen ist andererseits nicht in das Ghetto von Sondersituationen zu sperren, etwa in den nur fallweise stattfindenden Projektunterricht. Diskursives Lernen bedarf keiner zeitaufwendigen Unterrichtsvorhaben. Dies zu verdeutlichen dienen die beiden Unterrichtsbeispiele (Abb. 2a/b, 3).

Nicht nur die Schüler gilt es abzuholen, wo sie stehen - auch die Lehrer! Für diese ist diskursiver Unterricht oft sehr mühselig und verunsichernd. Nicht jeder hält die produktive Verunsicherung von Schülern aus, die es gewohnt sind, daß «zwei mal zwei vier ist», nicht jeder mag der Klage «Bei Ihnen ist es immer so anstrengend!» . . . und «Wann machen wir denn mal wieder richtige Erdkunde?» standzuhalten. Man braucht schon einen langen Atem.

Es gehört mehr dazu, als nur eine Betrachtungsalternative mitzubringen. Themen müssen so aufbereitet werden, daß ihre Anwendung möglich wird, im Gespräch zu Hause, bei der Auswahl von Fernsehsendungen. An geeigneten Themen besteht allerdings kein Mangel, z. B.: Naturkatastrophen - ein Ereignis des Klimas oder verfehlter Lebens- und Wirtschaftsweisen? Bevölkerungswachstum in der 3. Welt-Ursachen der Armut oder deren Folge? Not in Nord-Brasilien - Landreform oder Neulandgewinnung in Amazonien? Brandrodung - angepaßtes System oder Ursache der Umweltzerstörung? Staubstürme in den Great Plains - Fehlverhalten der Siedler oder Folge des Eisenbahnbaus? Entdeckungsreisen - Mutige Seefahrer oder Suche nach Gold und Gewürzen? Naturvölker - bewahren, sich selbst überlassen, oder sich selbst entwickeln lassen? Süd- afrika - Jedem das Seine oder gleiches Recht für alle? Fernurlaub in Kenia - Kulturzerstörung oder Völkerverständigung/Ghettobildung oder Entwicklung? Die Grenzen des Ackerbaus - von der Natur gezogen oder von der Rentabilität? Landschaftsgürtel in Afrika - Steuern sie die Verteilung der Vegetation oder auch der Armut?

\section{Literatur}

DAUM, E./SCHMIDT-WULFFEN, W. (1980): Erdkunde ohne Zukunft, Schöningh, Paderborn.

DER SPIEGEL (1988): "Die große Flut, die kommt erst noch» Nr. 14, S. $106 \mathrm{f}$.

FLECK, L. (1935/1980): Entstehung und Entwicklung einer wissenschaftlichen Tatsache. Benno Schwab, Berlin/Neuauflage Suhrkamp, Frankfurt.

FRANKFURTER RUNDSCHAU (1987): Tourismus als Landschaftsfresser vom 10.8 .

GRELL, J. (1984): Unterrichtspraxis. In: «betrifft erziehung», Nr. 8/9, S. 46-56.

HARD, G. (1982): Physisch-geographische Probleme im Unterricht. In: JANDER, L./SCHRAMKE, W.MENZEL, H.J. $(\mathrm{Hrg})$ : Metzler Handbuch für den Geographieunterricht, Metzler, Stuttgart, S. 173-289.

HELLBERGER, Ch. (1974): Marxismus als Methode. Fischer Athenäum, Frankfurt

MENSCHING, H. (1986): Die Sahelzone. Problemräume der Welt, H.6, Aulis/Deubner, Köln

MICHEHELLES, E. (1985): Das aktuelle politische Afrikabild in deutschen Schulbüchern, dargestellt und analysiert anhand des Faches Geographie. Dipl. Arbeit, maschinenschriftlich, Sozialwissenschaftliches Institut der Universität München

SCHMIDT-WULFFEN, W. (1982): Allgemeine Geographie. In: JANDER, L./SCHRAMKE, W. WENZEL, H.J., S. 15-20.

- (1985): Dürre und Hunger im Sahel. Gesellschaft und Natur. Urbs et Regio, Gesamthochschule Kassel, Bd. 37.

- (1986): Pluralismus für die Wissenschaft - Dogmatismus in der Schule? In: HUSA, K.NIELHABER, C.WOHLSCHLÄGL, H. (Hrg): Beiträge zur Didaktik der Geographie. Festschrift E. Troger, Bd. 2, Hirt, Wien, S. 47-62.

SCHULZ, W. (1984): Das Schulbuch, das Leitmedium? In: "betrifft erziehung", Nr. 8/9, S. 43-46

WERLEN, B. (1987): Gesellschaft, Handlung und Raum. Erdkundliches Wissen H. 89, Steiner, Stuttgart. 